\title{
Crystallization of Precise Side-Chain Giant Molecules with Tunable Sequences and Functionalities
}

Fengfeng Feng ${ }^{\mathrm{a}}$, Yu Shao ${ }^{\mathrm{b}}$, Wenjing Wu ${ }^{\mathrm{a}}$, Xiangqian Li ${ }^{\mathrm{a}}$, Chengyang Hong ${ }^{\mathrm{a}}$, Liang Jin ${ }^{\mathrm{a}}$, Kan Yue ${ }^{\mathrm{c}}$, Wen-Bin Zhang ${ }^{\mathrm{b}}$, Hao $\mathrm{Liu}^{\mathrm{a} *}$

aState Key Laboratory for Modification of Chemical Fibers and Polymer Materials, Center for Advanced LowDimension Materials, Donghua University, Shanghai 201620, China

bBeijing National Laboratory for Molecular Sciences, Key Laboratory of Polymer Chemistry \& Physics of Ministry of Education, Center for Soft Matter Science and Engineering, College of Chemistry and Molecular Engineering, Peking University, Beijing 100871, China

'South China Advanced Institute of Soft Matter Science and Technology, School of Molecular Science and Engineering, South China University of Technology, Guangzhou 510640, P. R. China

*Email: liuh@dhu.edu.cn 


\section{Contents}

1. Chemicals and Solvents.

2. Instrumentation.

3. Synthesis and Characterization.

4. 2D Crystal Preparation.

5. Supplementary Schemes, Tables and Figures.

Table S1. Giant molecules self-assembly solution system and corresponding concentration of the solution.

Scheme S1. Synthesis route of Mal-POM.

Scheme S2. Synthesis route of $\mathbf{B}_{2} V_{B_{2}}, \mathbf{B}_{3} V B$ and $\mathbf{B}_{\mathbf{4}} \mathbf{V}$.

Scheme S3. Synthesis route of $\mathbf{B}_{\mathbf{4}} \mathbf{A}, \mathbf{B}_{\mathbf{4}} \mathbf{D}$ and $\mathbf{B}_{\mathbf{4}} \mathbf{N}$.

Scheme S4. Synthesis route of $\mathbf{B}_{\mathbf{4}} \mathbf{P O M}$.

Figure S1. ${ }^{1} \mathrm{H}$ NMR spectra of Mal-VPOSS-ST, Mal-BPOSS-ST and Mal-POM.

Figure S2. ${ }^{1} \mathrm{H}$ NMR spectra of $\mathbf{B}_{2} \mathbf{A B}_{2}, \mathbf{B}_{2} \mathbf{V B}_{2}, \mathbf{B}_{3} \mathbf{A B}, \mathbf{B}_{3} \mathbf{V B}, \mathbf{B}_{4} A$ and $\mathbf{B}_{4} V$.

Figure S3. ${ }^{1} \mathrm{H}$ NMR spectra of $\mathbf{B}_{\mathbf{4}} \mathbf{N}, \mathbf{B}_{\mathbf{4}} \mathbf{D}$ and $\mathbf{B}_{\mathbf{4}} \mathbf{P O M}$.

Figure S4. MALDI-TOF MS of (a) $\mathbf{B}_{4}$, (b) $\mathbf{B}_{\mathbf{4}} \mathbf{V}$, (c) $\mathbf{B}_{3} \mathbf{V B}$ and (d) $\mathbf{B}_{2} \mathbf{V B}_{2}$.

Figure S5. TGA analysis of (a) $\mathbf{B}_{2} \mathbf{A} \mathbf{B}_{2}$, (b) $\mathbf{B}_{3} \mathbf{A B}$, (c) $\mathbf{B}_{\mathbf{4}} \mathbf{A}$, (d) $\mathbf{B}_{4} \mathbf{D}$, (e) $\mathbf{B}_{4} \mathbf{N}$ and (f) $\mathbf{B}_{4} \mathbf{P O M}$.

Figure S6. AFM images of giant molecules $\mathbf{B}_{\mathbf{2}} \mathbf{A} \mathbf{B}_{\mathbf{2}}, \mathbf{B}_{\mathbf{3}} \mathbf{A B}, \mathbf{B}_{\mathbf{4}} \mathbf{A}, \mathbf{B}_{\mathbf{4}} \mathbf{P O M}, \mathbf{B}_{\mathbf{4}} \mathbf{D}$ and $\mathbf{B}_{\mathbf{4}} \mathbf{N}$, (Scale bar $=500 \mathrm{~nm}$ ). The yellow arrow represents the base, and the green arrow represents the upper surface of the two-dimensional crystals. Figure S7. (a) [001] zone view of the crystal lattice model of T8 BPOSS, and (b) the corresponding simulated ED pattern with indices.

Figure S8. WAXD patterns of giant molecules $\mathbf{B}_{2} \mathbf{A B}_{2}, \mathbf{B}_{3} \mathbf{A B}, \mathbf{B}_{4} \mathbf{A}, \mathbf{B}_{\mathbf{4}} \mathbf{N}, \mathbf{B}_{\mathbf{4}} \mathbf{D}$ and $\mathbf{B}_{\mathbf{4}} \mathbf{P O M}$. 


\section{Chemicals and Solvents.}

Trifluoroacetic acid (TFA, 99\%+, Maclin), triethylsilane (TES, 99\%, Adamas), triethylamine (TEA, 99.5\%, J\&K Chemicals), N-Boc-ethylenediamine (99\%, Sigma-Aldrich), maleic anhydride (99\%, Sigma-Aldrich), acetic anhydride ( $>98 \%$, TCI America), sodium acetate trihydrate ( $>99.0 \%$, Sigma-Aldrich), Tin(II)chloride dihydrate ( $\geq 99.99 \%$, Sigma-Aldrich), acrylic acid ( $>99.0 \%$, TCI America), tetrabutylammonium bromide ( $\geq 98.0 \%$, Sigma-Aldrich), N-Ethoxycarbonyl-2-ethoxy-1,2-dihydroquinoline (99\%, Macklin), mercapto acetic acid (98\%, J\&k Chemicals), thioglycerol (97\%, Macklin), Sodium Tungstate Dihydrate $\left(\mathrm{Na}_{2} \mathrm{WO}_{4} \cdot 2 \mathrm{H}_{2} \mathrm{O}\right)$ $\left(98 \%+\right.$, Titan), Disodium hydrogen phosphate $\left(\mathrm{Na}_{2} \mathrm{HPO}_{4} \cdot 12 \mathrm{H}_{2} \mathrm{O}\right)$ (99.5\%, Sinopharm), 2-Hydroxy-4'-(2hydroxyethoxy)-2-methylpropiophenone (98.0\%, TCI America), cysteamine hydrochloride (>95\%, TCI America) were used as received. Dichloromethane (DCM), tetrahydrofuran (THF), chloroform, acetonitrile $(\mathrm{ACN})$, methanol $(\mathrm{MeOH})$, ether, acetone was used as received unless otherwise stated.

\section{Instrumentation and Characterization.}

Nuclear Magnetic Resonance (NMR). All ${ }^{1} \mathrm{H}$ NMR and ${ }^{13} \mathrm{C}$ NMR spectra were acquired in $\mathrm{CDCl}_{3}$ using a Bruker $400 \mathrm{MHz}$ NMR spectrometer. ${ }^{1} \mathrm{H}$ NMR spectra were referenced to the residual solvent peak in $\mathrm{CDCl}_{3}$ at $\delta 7.27 \mathrm{ppm}$, and ${ }^{13} \mathrm{C}$ NMR spectra were referenced to the residual peak in $\mathrm{CDCl}_{3}$ at $\delta 77.0 \mathrm{ppm}$.

Fourier-transform Infrared Spectroscopy (FTIR). Infrared spectra of the samples were recorded on a Bruker spectrometer (Vertex 70) by the potassium bromide ( $\mathrm{KBr}$ ) tableting method at room temperature. The data were processed using the Win-IR software.

Matrix-Assisted Laser Desorption/ionization Time-of-Flight (MALDI-TOF). MALDI-TOF mass spectroscopy (MS) were acquired on an UltrafleXtreme MALDI-TOF mass spectrometer (Bruker Daltonics) equipped with a $1 \mathrm{kHz}$ smart beam-II laser. Trans-2-[3-(4-tert-butyl-phenyl)-2-methyl-2-propenylidene]malononitrile (DCTB, Sigma-Aldrich, $>98 \%$ ) was used as the matrix and prepared in $\mathrm{CHCl}_{3}$ at a concentration of $20 \mathrm{mg} / \mathrm{mL}$. The cationizing agent sodium trifluoroacetate was prepared in ethanol at a concentration of 10 $\mathrm{mg} / \mathrm{mL}$. The matrix and cationizing salt solutions were mixed in a ratio of 10/1 (v/v). All samples were dissolved in $\mathrm{CHCl}_{3}$ at a concentration of $10 \mathrm{mg} / \mathrm{mL}$. After sample preparation and solvent evaporation, the target plate was inserted into the MALDI-TOF mass spectrometer. The attenuation of the laser was adjusted to minimize undesired fragmentation and maximize the sensitivity.

Thermogravimetric Analysis (TGA). Thermogravimetric analysis was carried out on a TA instrument (Discovery TGA 550) with a heating rate of $10{ }^{\circ} \mathrm{C} / \mathrm{min}$ in a nitrogen atmosphere. The nitrogen flow rate is 40 $\mathrm{mL} / \mathrm{min}$. The test temperature is from room temperature to $600{ }^{\circ} \mathrm{C}$.

Gel Permeation Chromatography (GPC). GPC was measured at $45^{\circ} \mathrm{C}$ on the ACQUITY APC instrument 
equipped with four ACQUITY APC XT columns (XT-45, XT-125, XT-200, XT-450 in series), a double flow type RI detector, and an ACQUITY APC TUV detector, using THF as eluent. The flow-rate was $0.6 \mathrm{~mL} / \mathrm{min}$. Data acquisition was performed using the Empower software, and molecular weights and molecular weight distributions were calibrated with polystyrene standards.

High Performance Liquid Chromatography (HPLC). HPLC data was analyzed at $70{ }^{\circ} \mathrm{C}$ on the Shanghai Wufeng LC-100 HP pump instrument equipped with Sepax BR-C18 column $(4.6 * 250 \mathrm{~mm}, 5 \mu \mathrm{m}, 300 \AA)$ produced from Sepax Technologies, using acetonitrile/tetrahydrofuran (65/35) as the eluent. The flow-rate was $0.6 \mathrm{~mL} / \mathrm{min}$. The detector adopts Speax HPLC-38 Refractive index detector. Data processing was accomplished using the software on a workstation equipped with V2008GPC.

Transmission Electron Microscopy (TEM). TEM measurements were conducted on a Thermo Scientific Talos F200S equipment with an accelerating voltage of $200 \mathrm{kV}$ to record the bright field images and SAED patterns. BF images were taken on a digital CCD camera and processed with the accessory digital imaging software. Selected-area election diffraction (SAED) were performed using a JEOL Digital System.

Atomic Force Microscopy (AFM). AFM experiments were performed on an Oxford Instruments MFP-3DBIO microscope to examine the $2 \mathrm{D}$ nanocrystals grown on the carbon-coated mica substrate by slow evaporation from its solution with a concentration of $0.2 \mathrm{mg} / \mathrm{mL}$. The images were taken in the tapping mode. The cantilever force was light enough to avoid any damage to the sample, yet strong enough so that the height features could be accurately explored. The scanning rate was $1.0 \mathrm{~Hz}$ for low-magnification images at a resolution of $512 \times 512$ pixels per image.

Small-angle X-ray scattering (SAXS). SAXS experiments were performed on Shanghai Synchrotron Radiation (SSRF), beamline BL16B1. The incident X-ray photon energy was $10 \mathrm{keV}$; the wavelength of the X-ray was $0.124 \mathrm{~nm}$; the photo flux was $1 \times 10^{11} \mathrm{phs} / \mathrm{s}$. The beam size is around $0.4 \times 0.5 \mathrm{~mm}^{2}$. Scattered Xrays were captured on a two-dimensional Pilatus detector. The instrument was calibrated with diffraction patterns from silver behenate. Some of the SAXS data was also recorded at Beamline 1W2A of the Beijing Synchrotron Radiation Facility (RSRF) with a wavelength of $1.54 \AA$. All the samples were vacuum dried for more than $24 \mathrm{~h}$ and then tested at room temperature.

Wide-angle X-ray diffraction (WAXD). WAXD data were recorded at Beamline 1W2A of the Beijing Synchrotron Radiation Facility (BSRF) with a wavelength of $1.54 \AA$. All the samples were vacuum dried for more than $24 \mathrm{~h}$ and then tested at room temperature. 


\section{Synthesis and characterization}

\subsection{Side-chain Giant Molecule Synthesis}

Ben-B $\mathbf{B}_{2} V-S T\left(\mathbf{B}_{2} \mathbf{V}\right)$. Ben-B-ST (B) $(200 \mathrm{mg}, 0.15 \mathrm{mmol})$ was dissolved in dried $\mathrm{CH}_{2} \mathrm{Cl}_{2}(5 \mathrm{~mL})$ in a Schlenk flask followed by dropwise addition of trifluoroacetic acid $(500 \mu \mathrm{L}, 6.7 \mathrm{mmol})$ inducing the solution to turn yellow instantly. After stirring for one minute at room temperature, TES was gradually added and the solution turned achromatic. The reaction solution $(1 \mathrm{~mL})$ was precipitated into methanol $(50 \mathrm{~mL})$ and collected by vacuum filtration giving Ben-B-SH as a white powder. Ben-B-SH (190 mg, $0.17 \mathrm{mmol})$ and Mal-B-ST (128 $\mathrm{mg}, 0.09 \mathrm{mmol})$ were added into a reaction flask followed by adding dried chloroform $(5 \mathrm{~mL})$ and one drop of triethylamine. The device was vigorously stirred at ambient temperature for $6 \mathrm{~h}$. The crude product was further purified by flash column chromatography silica gel (PE/EA =2:1 as eluent) to afford $\mathbf{B e n}-\mathbf{B}_{\mathbf{2}}-\mathbf{S T}\left(\mathbf{B}_{\mathbf{2}}\right)$ as a white solid. Yield: 81\%. In an oven-dried reaction flask with a magnetic stirrer, $\mathbf{B}_{\mathbf{2}}(200 \mathrm{mg}, 0.078 \mathrm{mmol})$ was fully dissolved in waterless DCM $(5 \mathrm{~mL})$. As trifluoroacetic acid ( $500 \mu \mathrm{L}, 6.7 \mathrm{mmol})$ was gradually added into the device, the mixed solution became yellow immediately. One minute later, TES was added dropwise and the solution turned colorless. Precipitation of the residue $(1 \mathrm{~mL})$ into frozen $\mathrm{MeOH}(50 \mathrm{~mL})$ followed by

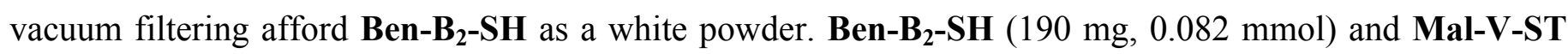
(104 mg, $0.09 \mathrm{mmol})$ were dissolved in dry $\mathrm{CHCl}_{3}(5 \mathrm{~mL})$ in an Schlenk flask followed by adding one drop of TEA via syringe. After stirring 6h under room temperature, the mixed solution was applied onto silica gel column and eluted with DCM/Methanol ( $\mathrm{v} / \mathrm{v}=59: 1)$ to yield the product $\mathbf{B}_{\mathbf{2}} \mathbf{V}$. The product was collected as a

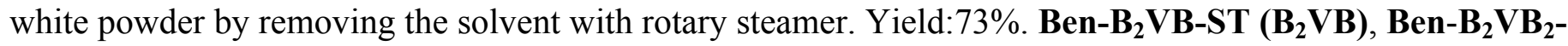
ST $\left(B_{2} V B_{2}\right)$, Ben-B ${ }_{3} V B-S T\left(B_{3} V B\right)$, Ben-B ${ }_{4} V-S T\left(B_{4} V\right)$ was obtained following similar experimental procedures described above.

$\mathbf{B}_{2}:{ }^{1} \mathrm{H}$ NMR $\left(400 \mathrm{MHz}, \mathrm{CDCl}_{3}, \mathrm{ppm}, \delta\right): 0.95\left(84 \mathrm{H},-\mathrm{CH}_{2}-\mathrm{CH}-\left(\mathrm{C}_{3}\right)_{2}\right), 0.64\left(28 \mathrm{H},-\mathrm{CH}_{2}-\mathrm{CH}-\left(\mathrm{CH}_{3}\right)_{2}\right), 1.87$ $\left(14 \mathrm{H},-\mathrm{CH}_{2}-\mathrm{C} \underline{\mathrm{H}}-\left(\mathrm{CH}_{3}\right)_{2}\right), 0.89\left(4 \mathrm{H},-\mathrm{Si}-\mathrm{C}_{2}-\mathrm{CH}_{2}-\right), 2.47\left(4 \mathrm{H},-\mathrm{Si}-\mathrm{CH}_{2}-\mathrm{C}_{2}-\right), 2.6\left(4 \mathrm{H},-\mathrm{S}-\mathrm{C}_{2}-\mathrm{CH}_{2}-\right), 4.2$ $\left(4 \mathrm{H},-\mathrm{S}-\mathrm{CH}_{2}-\underline{\mathrm{CH}}_{2}-\right), 4.64\left(4 \mathrm{H},-\underline{\mathrm{C}}_{2}-\mathrm{O}-\mathrm{CONH}-\mathrm{C}(-\mathrm{C}=\mathrm{O}) \underline{\mathrm{H}}-\right)$, $3.63\left(1 \mathrm{H},-\mathrm{S}-\mathrm{C}(-\mathrm{C}=\mathrm{O}) \underline{\mathrm{H}}-\mathrm{CH}_{2^{-}}\right), 3.04(4 \mathrm{H},-\mathrm{C}(-$ $\left.\mathrm{C}=\mathrm{O}) \mathrm{H}-\mathrm{CH}_{2}-\mathrm{S}-\right), 3.13\left(2 \mathrm{H},-\mathrm{C}(-\mathrm{C}=\mathrm{O}) \mathrm{H}-\underline{\mathrm{C}}_{2}-\mathrm{CO}-\right), 3.72\left(2 \mathrm{H},-\mathrm{C}_{2}-\mathrm{CH}_{2}-\mathrm{CONH}-\right), 2.45$ (2H, $-\mathrm{CH}_{2}-\mathrm{C}_{2}-$ CONH-), $7.87(2 \mathrm{H},-\mathrm{C}(=\mathrm{C} \underline{\mathrm{H}}) \mathrm{H}-\mathrm{C} \underline{\mathrm{H}}=) .{ }^{13} \mathrm{C} \mathrm{NMR}\left(100 \mathrm{MHz}, \mathrm{CDCl}_{3}, \mathrm{ppm}, \delta\right): 170.1,144.5,137.2,129.8,128.3$, 64.7, 51.7, 29.6, 26.1, 25.7, 23.4, 22.9, 13.2. FT-IR (KBr) v ( $\left.\mathrm{cm}^{-1}\right): 3457,2963,2875,1705,1538,1465,1402$, 1228, 1115 (Si-O-Si), 836, 745, 566, 483.

$\mathbf{B}_{2} \mathbf{V}:{ }^{1} \mathrm{H}$ NMR $\left(400 \mathrm{MHz}, \mathrm{CDCl}_{3}, \mathrm{ppm}, \delta\right): 0.95\left(84 \mathrm{H},-\mathrm{CH}_{2}-\mathrm{CH}-\left(\mathrm{C}_{3}\right)_{2}\right), 0.62\left(28 \mathrm{H},-\underline{\mathrm{C}}_{2}-\mathrm{CH}-\left(\mathrm{CH}_{3}\right)_{2}\right), 1.86$ $\left(14 \mathrm{H},-\mathrm{CH}_{2}-\mathrm{C} \underline{\mathrm{H}}-\left(\mathrm{CH}_{3}\right)_{2}\right), 5.9\left(21 \mathrm{H},-\mathrm{C} \underline{\mathrm{H}}=\mathrm{C}_{2}\right), 2.58\left(6 \mathrm{H},-\mathrm{Si}-\mathrm{CH}_{2}-\underline{\mathrm{CH}}_{2}-\right), 2.75\left(6 \mathrm{H},-\mathrm{S}-\mathrm{C}_{2}-\mathrm{CH}_{2}-\right), 4.27(6 \mathrm{H}$, $\left.-\mathrm{S}-\mathrm{CH}_{2}-\mathrm{C}_{2}-\right), 4.79(3 \mathrm{H},-\mathrm{CONH}-\mathrm{C}(-\mathrm{C}=\mathrm{O}) \underline{\mathrm{H}}-), 3.13\left(6 \mathrm{H},-\mathrm{C}(-\mathrm{C}=\mathrm{O}) \mathrm{H}-\mathrm{C}_{2}-\mathrm{S}-\right), 3.81(6 \mathrm{H},-\mathrm{S}-\mathrm{C}(\mathrm{C}=\mathrm{O}) \underline{\mathrm{H}}-$ $\left.\mathrm{CH}_{2^{-}},-\underline{\mathrm{H}}_{2}-\mathrm{CH}_{2}-\mathrm{CONH}-\right), 2.43\left(4 \mathrm{H},-\mathrm{CH}_{2}-\underline{\mathrm{C}}_{2}-\mathrm{CONH}-\right), 7.87(2 \mathrm{H},-\mathrm{C}(=\mathrm{C} \underline{\mathrm{H}}) \mathrm{H}-\mathrm{C} \underline{\mathrm{H}}=) .{ }^{13} \mathrm{C} \mathrm{NMR}(100 \mathrm{MHz}$, 
$\left.\mathrm{CDCl}_{3}, \mathrm{ppm}, \delta\right): 170.4,144.3,137.2,129.5,128.6,64.5,51.4,29.3,26.5,25.5,23.2,22.1,13.2 . \mathrm{FT}-\mathrm{IR}(\mathrm{KBr})$ $v\left(\mathrm{~cm}^{-1}\right): 3462,2955,1705,1536,1468,1402,1225,1113$ (Si-O-Si), 838, 746, 564, 486.

$\mathbf{B}_{2}$ VB: ${ }^{1} \mathrm{H}$ NMR (400 MHz, $\left.\mathrm{CDCl}_{3}, \mathrm{ppm}, \delta\right): 0.95\left(126 \mathrm{H},-\mathrm{CH}_{2}-\mathrm{CH}-\left(\mathrm{CH}_{3}\right)_{2}\right), 0.62\left(42 \mathrm{H},-\underline{\mathrm{C}}_{2}-\mathrm{CH}-\left(\mathrm{CH}_{3}\right)_{2}\right)$, $1.86\left(21 \mathrm{H},-\mathrm{CH}_{2}-\mathrm{C} \underline{H}-\left(\mathrm{CH}_{3}\right)_{2}\right), 5.9\left(21 \mathrm{H},-\mathrm{C} \underline{H}=\mathrm{C}_{2}\right), 2.58\left(8 \mathrm{H},-\mathrm{Si}_{-} \mathrm{CH}_{2}-\mathrm{C}_{2}-\right), 2.75\left(8 \mathrm{H},-\mathrm{S}-\mathrm{C}_{2}-\mathrm{CH}_{2}-\right), 4.27$ $\left(8 \mathrm{H},-\mathrm{S}-\mathrm{CH}_{2}-\underline{\mathrm{C}}_{2}-\right), 4.79(4 \mathrm{H},-\mathrm{CONH}-\mathrm{C}(-\mathrm{C}=\mathrm{O}) \underline{\mathrm{H}}-), 3.13\left(8 \mathrm{H},-\mathrm{C}(-\mathrm{C}=\mathrm{O}) \mathrm{H}-\underline{\mathrm{C}}_{2}-\mathrm{S}-\right), 3.81(8 \mathrm{H},-\mathrm{S}-\mathrm{C}(\mathrm{C}=\mathrm{O}) \underline{\mathrm{H}}-$ $\left.\mathrm{CH}_{2}-,-\underline{\mathrm{H}}_{2}-\mathrm{CH}_{2}-\mathrm{CONH}-\right), 2.43\left(6 \mathrm{H},-\mathrm{CH}_{2}-\underline{\mathrm{C}}_{2}-\mathrm{CONH}-\right), 7.87(2 \mathrm{H},-\mathrm{C}(=\mathrm{C} \underline{\mathrm{H}}) \mathrm{H}-\mathrm{C} \underline{\mathrm{H}}=)$ ). ${ }^{13} \mathrm{C} \mathrm{NMR}(100 \mathrm{MHz}$, $\left.\mathrm{CDCl}_{3}, \mathrm{ppm}, \delta\right): 170.2,144.5,137.6,129.7,128.4,64.6,51.6,29.3,26.2,25.8,23.2,22.5,13.2 . \mathrm{FT}-\mathrm{IR}(\mathrm{KBr})$ $v\left(\mathrm{~cm}^{-1}\right):$ 3457, 2952, 1702, 1534, 1465, 1407, 1222, 1116 (Si-O-Si), 841, 744, 567, 484.

$\mathbf{B}_{2} \mathbf{V B}_{2}:{ }^{1} \mathrm{H}$ NMR $\left(400 \mathrm{MHz}, \mathrm{CDCl}_{3}, \mathrm{ppm}, \delta\right): 0.95\left(168 \mathrm{H},-\mathrm{CH}_{2}-\mathrm{CH}-\left(\mathrm{C}_{3}\right)_{2}\right), 0.62\left(56 \mathrm{H},-\underline{\mathrm{C}}_{2}-\mathrm{CH}-\left(\mathrm{CH}_{3}\right)_{2}\right)$, $1.86\left(28 \mathrm{H},-\mathrm{CH}_{2}-\mathrm{C} \underline{\mathrm{H}}-\left(\mathrm{CH}_{3}\right)_{2}\right), 5.9\left(21 \mathrm{H},-\mathrm{C} \underline{\mathrm{H}}=\mathrm{C}_{2}\right), 2.58\left(10 \mathrm{H},-\mathrm{Si}_{2}-\mathrm{CH}_{2}-\mathrm{CH}_{2}-\right), 2.75\left(10 \mathrm{H},-\mathrm{S}^{-} \underline{\mathrm{C}}_{2}-\mathrm{CH}_{2}-\right)$, $4.27\left(10 \mathrm{H},-\mathrm{S}-\mathrm{CH}_{2}-\underline{\mathrm{C}}_{2}-\right), 4.79(5 \mathrm{H},-\mathrm{CONH}-\mathrm{C}(-\mathrm{C}=\mathrm{O}) \underline{\mathrm{H}}-), 3.13\left(10 \mathrm{H},-\mathrm{C}(-\mathrm{C}=\mathrm{O}) \mathrm{H}-\underline{\mathrm{C}}_{2}-\mathrm{S}-\right), 3.81(10 \mathrm{H},-\mathrm{S}-$ $\left.\mathrm{C}(\mathrm{C}=\mathrm{O}) \underline{\mathrm{H}}-\mathrm{CH}_{2}-,-\mathrm{C}_{2}-\mathrm{CH}_{2}-\mathrm{CONH}-\right), 2.43\left(8 \mathrm{H},-\mathrm{CH}_{2}-\mathrm{C}_{2}-\mathrm{CONH}-\right), 7.87(2 \mathrm{H},-\mathrm{C}(=\mathrm{C} \underline{\mathrm{H}}) \mathrm{H}-\mathrm{C} \underline{\mathrm{H}}=) .{ }^{13} \mathrm{C}$ NMR

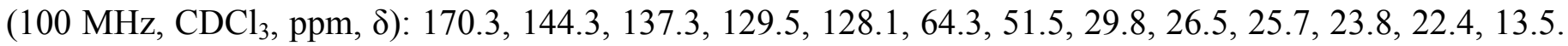
FT-IR (KBr) v $\left(\mathrm{cm}^{-1}\right)$ : 3459, 2960, 1708, 1540, 1467, 1405, 1226, 1114 (Si-O-Si), 838, 748, 566, 487.

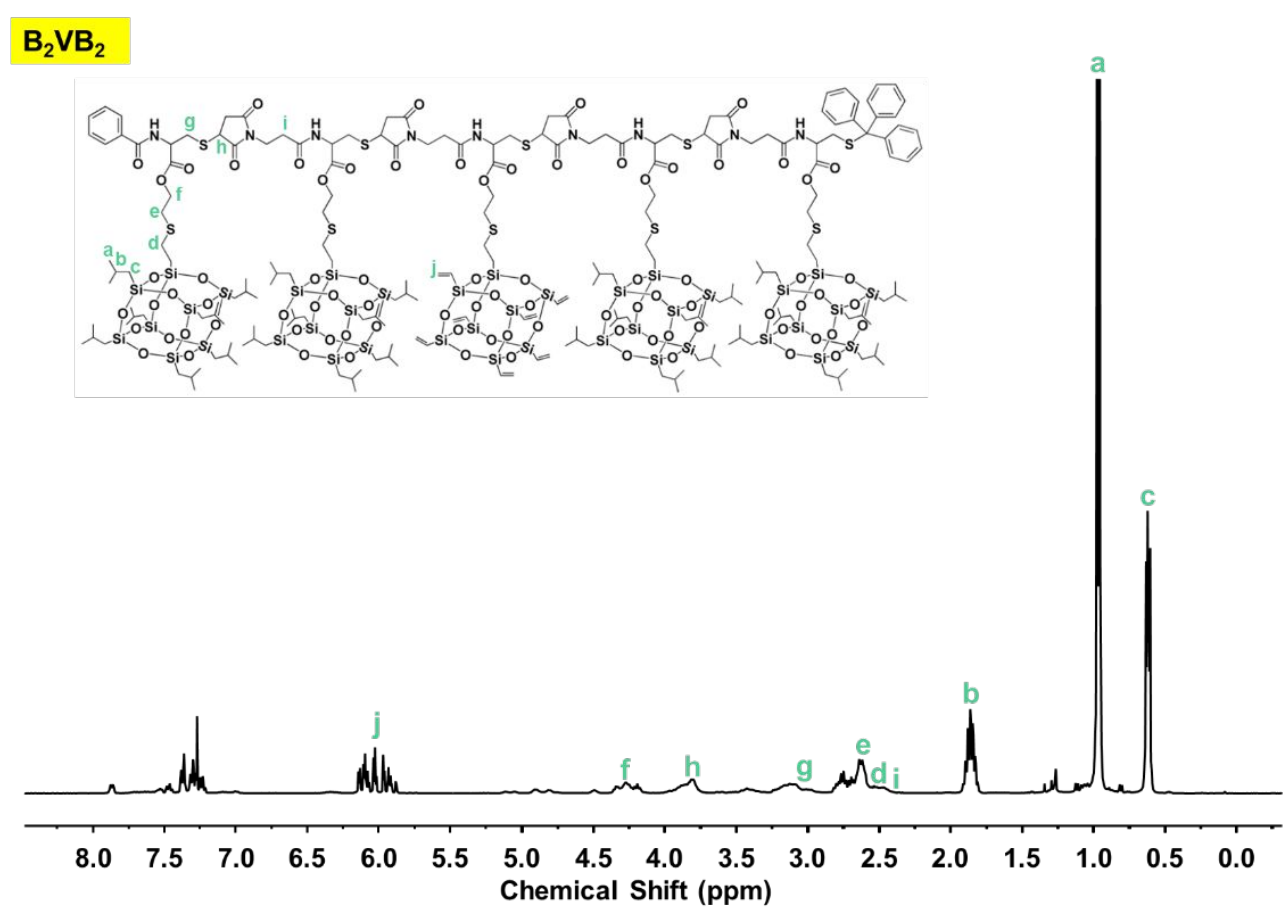

$\mathbf{B}_{3}$ VB: ${ }^{1} \mathrm{H}$ NMR $\left(400 \mathrm{MHz}, \mathrm{CDCl}_{3}, \mathrm{ppm}, \delta\right): 0.93\left(168 \mathrm{H},-\mathrm{CH}_{2}-\mathrm{CH}-\left(\mathrm{CH}_{3}\right)_{2}\right), 0.61\left(56 \mathrm{H},-\underline{\mathrm{C}}_{2}-\mathrm{CH}-\left(\mathrm{CH}_{3}\right)_{2}\right)$, $1.83\left(28 \mathrm{H},-\mathrm{CH}_{2}-\mathrm{C} \underline{\mathrm{H}}-\left(\mathrm{CH}_{3}\right)_{2}\right), 5.87\left(21 \mathrm{H},-\mathrm{C} \underline{\mathrm{H}}=\mathrm{C}_{2}\right), 2.55\left(10 \mathrm{H},-\mathrm{Si}^{-} \mathrm{CH}_{2}-\mathrm{C}_{2}{ }^{-}\right), 2.71\left(10 \mathrm{H},-\mathrm{S}^{-} \underline{\mathrm{H}}_{2}-\mathrm{CH}_{2}-\right)$, $4.24\left(10 \mathrm{H},-\mathrm{S}-\mathrm{CH}_{2}-\underline{\mathrm{C}}_{2}-\right), 4.73(5 \mathrm{H},-\mathrm{CONH}-\mathrm{C}(-\mathrm{C}=\mathrm{O}) \underline{\mathrm{H}}-), 3.11\left(10 \mathrm{H},-\mathrm{C}(-\mathrm{C}=\mathrm{O}) \mathrm{H}-\underline{\mathrm{C}}_{2}-\mathrm{S}-\right), 3.77(10 \mathrm{H},-\mathrm{S}-$ $\left.\mathrm{C}(\mathrm{C}=\mathrm{O}) \underline{\mathrm{H}}-\mathrm{CH}_{2}-,-\mathrm{CH}_{2}-\mathrm{CH}_{2}-\mathrm{CONH}-\right), 2.41\left(8 \mathrm{H},-\mathrm{CH}_{2}-\underline{\mathrm{H}}_{2}-\mathrm{CONH}-\right), 7.85(2 \mathrm{H},-\mathrm{C}(=\mathrm{C} \underline{\mathrm{H}}) \mathrm{H}-\mathrm{C} \underline{\mathrm{H}}=) .{ }^{13} \mathrm{C}$ NMR

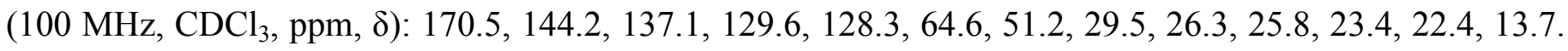
FT-IR (KBr) v (cm-1): 3457, 2964, 1711, 1543, 1468, 1409, 1225, 1118 (Si-O-Si), 833, 745, 564, 485. 


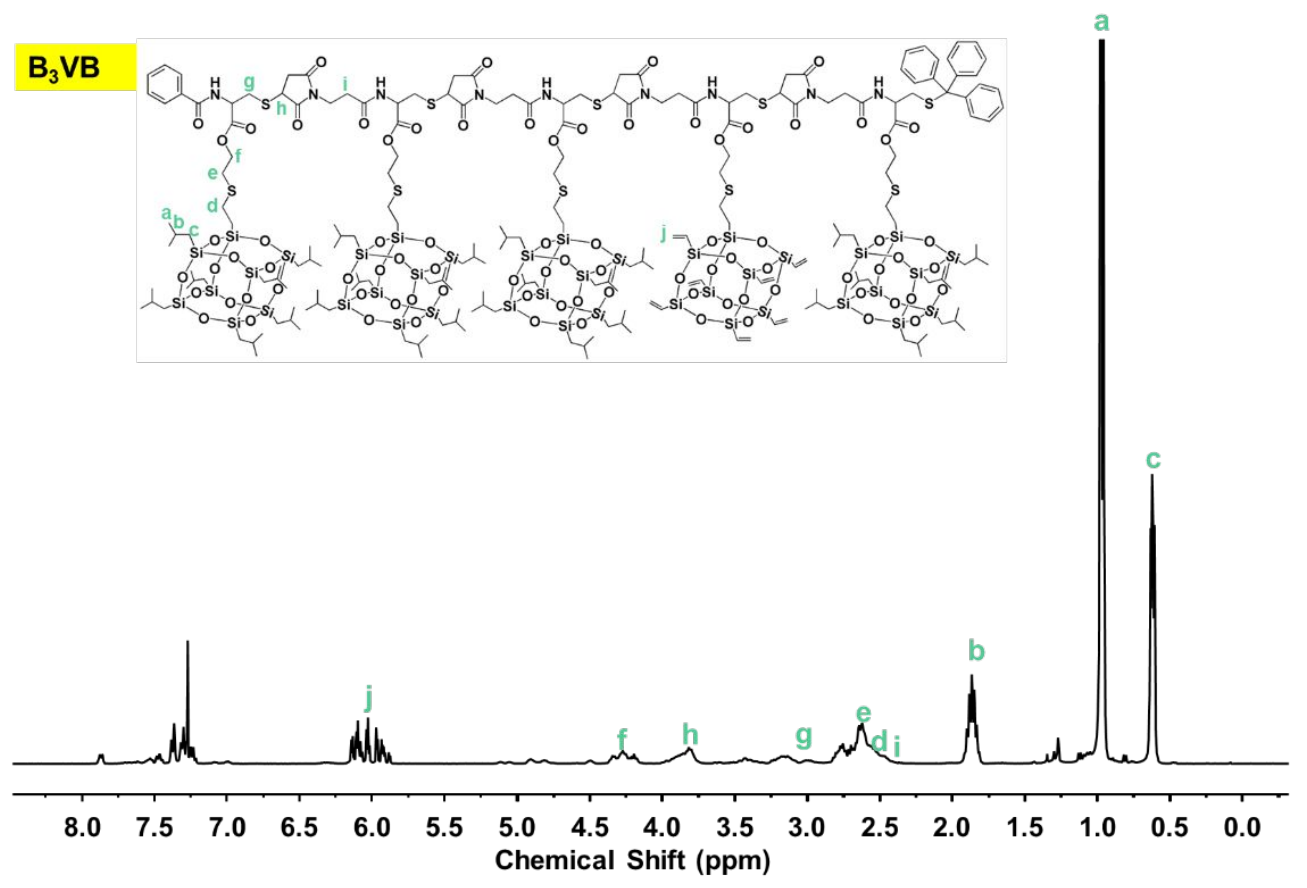

$\mathbf{B}_{4} \mathbf{V}:{ }^{1} \mathrm{H}$ NMR (400 MHz, $\left.\mathrm{CDCl}_{3}, \mathrm{ppm}, \delta\right): 0.96\left(168 \mathrm{H},-\mathrm{CH}_{2}-\mathrm{CH}-\left(\mathrm{C}_{3}\right)_{2}\right), 0.64\left(56 \mathrm{H},-\underline{\mathrm{H}}_{2}-\mathrm{CH}-\left(\mathrm{CH}_{3}\right)_{2}\right), 1.85$ $\left(28 \mathrm{H},-\mathrm{CH}_{2}-\mathrm{C} \underline{\mathrm{H}}-\left(\mathrm{CH}_{3}\right)_{2}\right), 5.88\left(21 \mathrm{H},-\mathrm{C} \underline{\mathrm{H}}=\mathrm{C}_{2}\right), 2.57\left(10 \mathrm{H},-\mathrm{Si}-\mathrm{CH}_{2}-\underline{\mathrm{C}}_{2}-\right), 2.77\left(10 \mathrm{H},-\mathrm{S}-\mathrm{C}_{2}-\mathrm{CH}_{2}-\right), 4.26$ $\left(10 \mathrm{H},-\mathrm{S}-\mathrm{CH}_{2}-\underline{\mathrm{C}}_{2}-\right), 4.81(5 \mathrm{H},-\mathrm{CONH}-\mathrm{C}(-\mathrm{C}=\mathrm{O}) \underline{\mathrm{H}}-), 3.14\left(10 \mathrm{H},-\mathrm{C}(-\mathrm{C}=\mathrm{O}) \mathrm{H}-\underline{\mathrm{C}}_{2}-\mathrm{S}-\right), 3.74(10 \mathrm{H},-\mathrm{S}-$ $\left.\mathrm{C}(\mathrm{C}=\mathrm{O}) \underline{\mathrm{H}}-\mathrm{CH}_{2}-,-\mathrm{C}_{2}-\mathrm{CH}_{2}-\mathrm{CONH}-\right), 2.44\left(8 \mathrm{H},-\mathrm{CH}_{2}-\mathrm{C}_{2}-\mathrm{CONH}-\right), 7.86(2 \mathrm{H},-\mathrm{C}(=\mathrm{C} \underline{\mathrm{H}}) \mathrm{H}-\mathrm{C} \underline{\mathrm{H}}=) .{ }^{13} \mathrm{C}$ NMR (100 MHz, $\mathrm{CDCl}_{3}$, ppm, $\delta$ ): 170.8 143.4, 137.7, 129.2, 127.8, 65.2, 51.6, 29.8, 26.7, 25.6, 23.7, 22.1, 13.8. FT-IR (KBr) v (cm-1): 3456, 2965, 1714, 1545, 1466, 1407, 1226, 1116 (Si-O-Si), 831, 747, 562, 485.

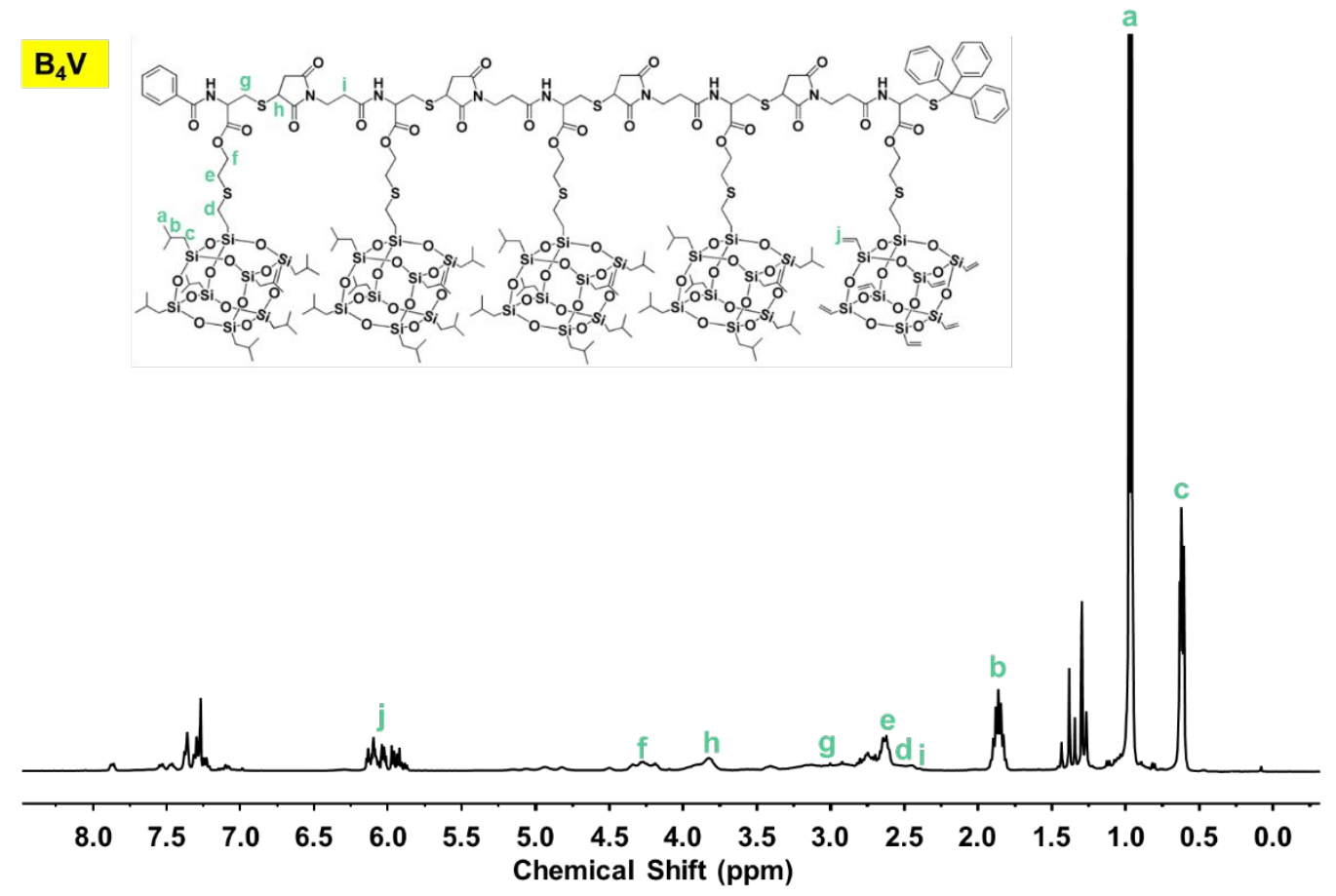

Mal-POM. Mal- $\mathrm{NH}_{2}$ was prepared following the previously reported procedures ${ }^{[1]}$, N-Boc-ethylenediamine (10 mmol, in $\mathrm{CH}_{2} \mathrm{Cl}_{2}$ ) was added dropwise to maleic anhydride (10 mmol, in $\mathrm{CH}_{2} \mathrm{Cl}_{2}$ ) under nitrogen and then stirred at room temperature for two hours to obtain the intermediate product 1 . Acetic anhydride $(74.5 \mathrm{mmol})$ was poured into the mixture that contains the filtered intermediate product $(10 \mathrm{mmol})$, triethylamine $(11.5$ 
$\mathrm{mmol})$, and sodium acetate trihydrate $(11 \mathrm{mmol})$ in acetone $(20 \mathrm{~mL})$ under nitrogen and stirred at $343 \mathrm{~K}$ for 3 hours. The addition of cold water $(20 \mathrm{~mL})$ resulted in a precipitate, which was further purified as the intermediate product 2 using column chromatography. The product $2(5 \mathrm{mmol})$ was dissolved in $\mathrm{CH}_{2} \mathrm{Cl}_{2}(20$ $\mathrm{mL})$ and stirred with the injected $\mathrm{CF}_{3} \mathrm{COOH}(5 \mathrm{mmol})$ for 3 hours at room temperature to get the product Mal$\mathrm{NH}_{2}$. EEDQ (196.2 mg, $\left.0.79 \mathrm{mmol}\right)$ was dissolved in a round bottom flask with acetonitrile (40 mL) followed by adding (TBA) $)_{4} \alpha-\left[\mathrm{PW}_{11} \mathrm{O}_{39} \mathrm{SnCH}_{2} \mathrm{CH}_{2} \mathrm{COOH}\right]$ (prepared following the previously reported procedures ${ }^{[2]}$ ) ( $2 \mathrm{~g}, 0.53 \mathrm{mmol})$. The reaction mixture was stirred in an oil bath at $90^{\circ} \mathrm{C}$ for 15 minutes, and then $\mathrm{Mal}^{-\mathrm{NH}_{2}}$ (223 mg, $1.59 \mathrm{mmol}$ ) was added. After reacting for overnight, the crude solution was purified by vacuum filtration and the filtered solution was dried by the rotary steamer. The product was precipitated into ether for further purification. Yield: 56\%. ${ }^{1} \mathrm{H} \mathrm{NMR}\left(400 \mathrm{MHz}, \mathrm{CDCl}_{3}, \mathrm{ppm}, \delta\right): 1.02\left(48 \mathrm{H}, \mathrm{C}_{3}-\mathrm{CH}_{2}-\mathrm{CH}_{2}-\right), 1.42(32 \mathrm{H}$, $\left.\mathrm{CH}_{3}-\mathrm{C}_{2}-\mathrm{CH}_{2}-\right), 1.66\left(32 \mathrm{H}, \mathrm{CH}_{3}-\mathrm{CH}_{2}-\mathrm{C}_{2}-\right), 3.17\left(32 \mathrm{H}, \mathrm{CH}_{3}-\mathrm{CH}_{2}-\mathrm{CH}_{2}-\mathrm{C}_{2}-\right), 3.34$ (2H, - $\left.\mathrm{CH}_{2}-\mathrm{CH}_{2}-\mathrm{CONH}-\right)$, $3.57\left(2 \mathrm{H},-\underline{\mathrm{C}}_{2}-\mathrm{CH}_{2}-\mathrm{CONH}-\right), 6.79$ (2H, $\left.-\mathrm{CO}-\mathrm{C} \underline{\mathrm{H}}=\mathrm{C} \underline{\mathrm{H}}-\mathrm{CO}-\right) .{ }^{13} \mathrm{C} \mathrm{NMR}\left(100 \mathrm{MHz}, \mathrm{CDCl}_{3}, \mathrm{ppm}, \delta\right): 163$, 135.6, 62.5, 42.7, 41.2, 26.3, 21.3, 13.4. FT-IR (KBr) v (cm-1): 3444, 2964, 2871, 1706, 1643, 1479, 1378, $1068,964,885,804,590,511$.

Ben-B ${ }_{4}$ POM-ST (B $\mathbf{B}_{4}$ POM). B $\mathbf{B}_{4}(150 \mathrm{mg}, 0.03 \mathrm{mmol})$ was completely dissolved in a round-bottom flask equipped with a magnetic stirrer. Trifluoroacetic acid $(500 \mu \mathrm{L}, 6.7 \mathrm{mmol})$ was added dropwise via a pipette gun into the solution inducing the reaction mixture become yellow immediately. Stirring for one minute, TES was gradually added until the solution turned colorless. Precipitation of the residue $(1 \mathrm{~mL})$ into frozen $\mathrm{MeOH}$

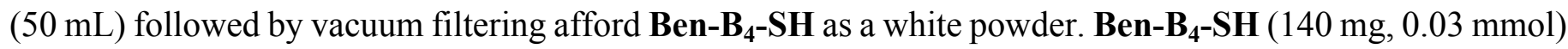
dissolved in THF (2 mL) and Mal-POM $(192 \mathrm{mg}, 0.06 \mathrm{mmol})$ dissolved in acetonitrile $(2 \mathrm{~mL})$ were mixed in an oven-dried reaction flask followed by the adding two drops of TEA. The mixture was stirred overnight at ambient temperature, and then dried by the rotary steamer. The crude product was further dissolved in DCM $(10 \mathrm{~mL})$ followed by vacuum filtration to remove solid particles. The filtered solution $(1 \mathrm{~mL})$ was precipitated into methanol $(50 \mathrm{~mL})$ and collected by vacuum filtration giving $\mathbf{B}_{\mathbf{4}} \mathbf{P O M}$ as a pale yellow solid. Yield:48\%. ${ }^{1} \mathrm{H}$ NMR (400 MHz, $\left.\mathrm{CDCl}_{3}, \mathrm{ppm}, \delta\right): 0.95\left(216 \mathrm{H},-\mathrm{CH}_{2}-\mathrm{CH}-\left(\mathrm{CH}_{3}\right)_{2}\right), 0.61\left(56 \mathrm{H},-\mathrm{C}_{2}-\mathrm{CH}-\left(\mathrm{CH}_{3}\right)_{2}\right), 1.85(28 \mathrm{H}$, $\left.-\mathrm{CH}_{2}-\mathrm{C} \underline{\mathrm{H}}-\left(\mathrm{CH}_{3}\right)_{2}\right), 1.40\left(32 \mathrm{H}, \mathrm{CH}_{3}-\mathrm{C}_{2}-\mathrm{CH}_{2}-\right), 1.71\left(32 \mathrm{H}, \mathrm{CH}_{3}-\mathrm{CH}_{2}-\mathrm{C}_{2}-\mathrm{CH}_{2}-\right), 3.28\left(32 \mathrm{H}, \mathrm{CH}_{3}-\mathrm{CH}_{2}-\mathrm{CH}_{2}-\right.$ $\left.\mathrm{C}_{2^{-}}\right), 2.61\left(8 \mathrm{H},-\mathrm{Si}-\mathrm{CH}_{2}-\underline{\mathrm{CH}}_{2}\right), 2.76\left(8 \mathrm{H},-\mathrm{S}-\mathrm{C}_{2}-\mathrm{CH}_{2}-\right), 4.24\left(8 \mathrm{H},-\mathrm{S}-\mathrm{CH}_{2}-\underline{\mathrm{C}}_{2}{ }^{-}\right), 4.82(4 \mathrm{H},-\mathrm{CONH}-\mathrm{C}(-$ $\mathrm{C}=\mathrm{O}) \underline{\mathrm{H}}-), 3.14\left(8 \mathrm{H},-\mathrm{C}(-\mathrm{C}=\mathrm{O}) \mathrm{H}-\mathrm{C}_{2}-\mathrm{S}-\right), 3.74\left(12 \mathrm{H},-\mathrm{S}-\mathrm{C}(\mathrm{C}=\mathrm{O}) \mathrm{H}-\mathrm{CH}_{2-},-\mathrm{C}_{2}-\mathrm{CH}_{2}-\mathrm{CONH}-\right), 2.44$ (6H, $\left.\mathrm{CH}_{2}-\underline{\mathrm{C}}_{2}-\mathrm{CONH}-\right), 7.86(2 \mathrm{H},-\mathrm{C}(=\mathrm{C} \underline{\mathrm{H}}) \mathrm{H}-\mathrm{C} \underline{\mathrm{H}}=) .{ }^{13} \mathrm{C} \mathrm{NMR}\left(100 \mathrm{MHz}, \mathrm{CDCl}_{3}, \mathrm{ppm}, \delta\right): 170.6,144.5,137.3$, 129.1, 128.6, 64.8, 61.5, 51.5, 29.4, 26.5, 25.4, 23.2, 22.6, 13.4. FT-IR (KBr) v (cm-1): 3451, 2958, 2861, 1704, 1539, 1464, 1405, 1223, 1114 (Si-O-Si), 828, 742, 557, 479. 


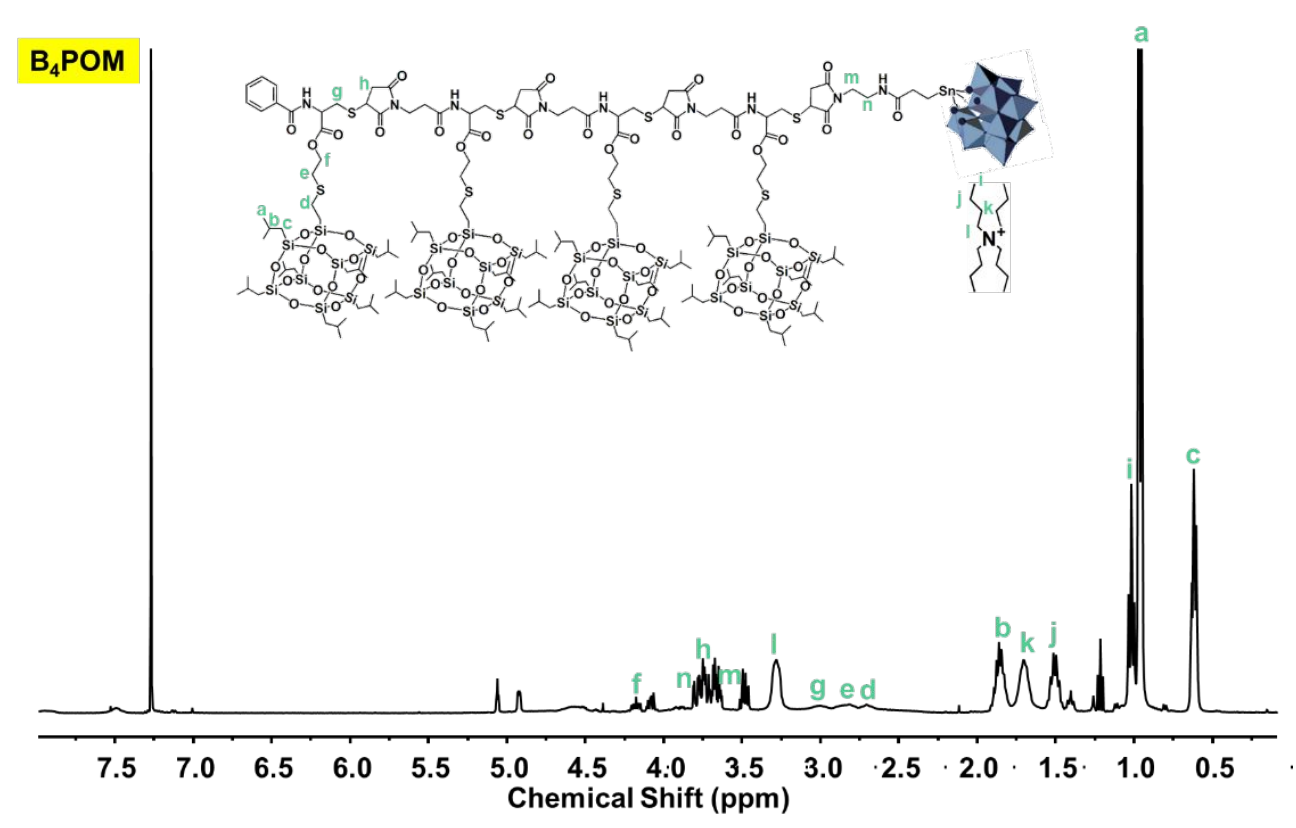

\subsection{General preparation process for samples $B_{2} A B_{2}, B_{3} A B, B_{4} A$.}

Taking $\mathbf{B}_{2} \mathbf{A B}_{2}$ as an example. $\mathbf{B}_{2} \mathbf{V} \mathbf{B}_{2}(50 \mathrm{mg}, 0.009 \mathrm{mmol})$ was added into a reaction vessel with waterless THF ( $5 \mathrm{~mL}$ ) followed by adding amount of 2-Hydroxy-4'-(2-hydroxyethoxy)-2-methylpropiophenone at room temperature. Mercapto acetic acid $(51 \mu \mathrm{L}, 0.72 \mathrm{mmol})$ was added gradually via a syringe. The mixed solution was stirred and illuminated under $362 \mathrm{~nm}$ UV light for $50 \mathrm{~min}$. Then precipitation of $1 \mathrm{~mL}$ of the resulting concentrated solution into $50 \mathrm{~mL}$ frozen methanol followed by vacuum filtering gave product as a white powder with removal of solvent in vacuum.

\subsection{General preparation process for sample $\mathrm{B}_{\mathbf{4}} \mathrm{D}$.}

In an oven-dried reaction vessel, $\mathbf{B}_{4} \mathbf{V}(65 \mathrm{mg}, 0.011 \mathrm{mmol})$ and a catalytic amount of 2-Hydroxy-4'-(2hydroxyethoxy)-2-methylpropiophenone were fully dissolved in dried THF ( $5 \mathrm{~mL})$. Thioglycerol $(48 \mu \mathrm{L}, 0.55$ mmol) was added dropwise via a syringe. The mixed solution was stirred and illuminated under $362 \mathrm{~nm}$ UV light for $50 \mathrm{~min}$. Then precipitation of $1 \mathrm{~mL}$ of the resulting crude solution into $50 \mathrm{~mL}$ frozen methanol followed by vacuum filtering afford product as a white powder with removal of solvent in vacuum.

\subsection{General preparation process for sample $B_{4} N$.}

In an oven-dried reaction flask with a magnetic stirrer, $\mathbf{B}_{\mathbf{4}} \mathbf{V}(65 \mathrm{mg}, 0.011 \mathrm{mmol})$, cysteamine hydrochloride (66 mg, $0.55 \mathrm{mmol}$ ) and a catalytic amount of 2-Hydroxy-4'-(2-hydroxyethoxy)-2-methylpropiophenone were fully dissolved in THF ( $5 \mathrm{~mL}$ ). The mixed solution was stirred and illuminated under $362 \mathrm{~nm}$ UV light for 50 min. Then precipitation of $1 \mathrm{~mL}$ of the resulting crude solution into $50 \mathrm{~mL}$ frozen methanol followed by vacuum filtering afford product as a white powder with removal of solvent in vacuum. 


\section{2D Crystal Preparation.}

Giant molecules $\mathbf{B}_{2} \mathbf{A} \mathbf{B}_{2}, \mathbf{B}_{\mathbf{3}} \mathbf{A B}, \mathbf{B}_{\mathbf{4}} \mathbf{A}$ and $\mathbf{B}_{\mathbf{4}} \mathbf{N}, \mathbf{B}_{\mathbf{4}} \mathbf{D}$ and $\mathbf{B}_{\mathbf{4}} \mathbf{P O M}$ were respectively dissolved in THF to give a clear solution with concentration of $1 \mathrm{mg} / \mathrm{mL} .200 \mu \mathrm{L}$ of the giant molecule solution was pipetted to a $2 \mathrm{~mL}$ glass vial, then DMF was injected into the solution to reach different solvent ratios, details are shown in the table S1 blow. After the completion of injecting process, the solution with different solvent ratios were sealed and placed in $25^{\circ} \mathrm{C}$ oven for $24 \mathrm{~h}$. Crystal samples for TEM experiments were grown by dropping of its THF solution with corresponding concentration on a carbon-coated mica substrate by carefully diffusing water into the carbon-mica interface. The floating carbon film was then picked up by copper grids for TEM experiments. Samples for AFM measurements were prepared by the same way. Powder crystals for SAXS and WAXD measurements were obtained by slow diffusion of methanol vapor into its $5 \mathrm{mg} / \mathrm{mL}$ chloroform solution in a sealed chamber. The white crystals were collected through high-speed centrifuge, dried in vacuum, and directly used for the X-ray tests.

Table S1. Giant molecules self-assembly solution system and corresponding concentration of the solution.

\begin{tabular}{ccc:ccc}
\hline Sample & THF:DMF & $\mathbf{C}(\mathbf{m g} / \mathbf{m L})$ & Sample & THF:DMF & $\mathbf{C}(\mathbf{m g} / \mathbf{m L})$ \\
\hdashline $\mathbf{B}_{2} \mathbf{A B} \mathbf{B}_{2}$ & $1: 2$ & 0.2 & $\mathbf{B}_{\mathbf{4}} \mathbf{N}$ & $1: 1$ & 0.2 \\
$\mathbf{B}_{3} \mathbf{A B}$ & $1: 2$ & 0.2 & $\mathbf{B}_{4} \mathbf{D}$ & $1: 2$ & 0.2 \\
$\mathbf{B}_{4} \mathbf{A}$ & $1: 2$ & 0.2 & $\mathbf{B}_{4} \mathbf{P O M}$ & $1: 2$ & 0.2 \\
\hline
\end{tabular}

\section{Supplementary Schemes, Tables and Figures.}

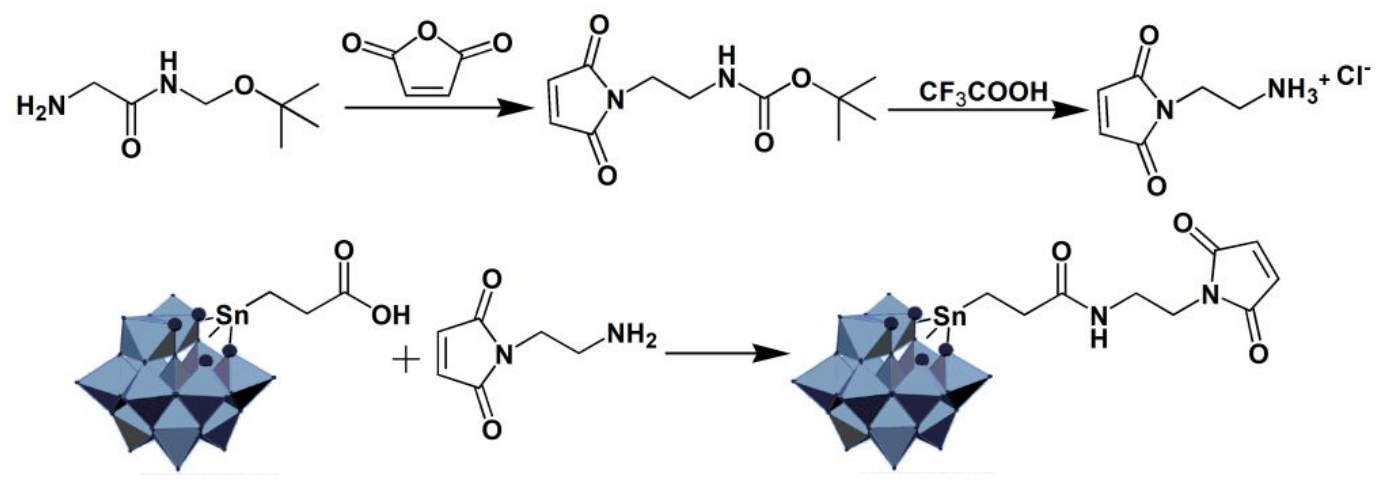

Scheme S1. Synthesis route of Mal-POM. 


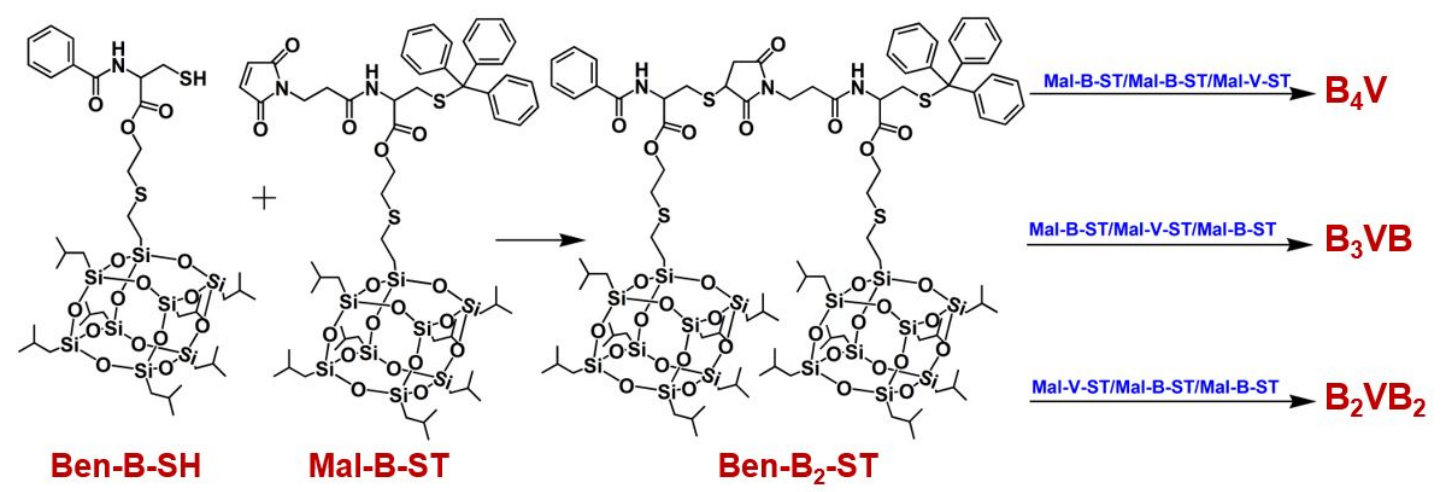

Scheme S2. Synthesis route of $\mathbf{B}_{2} V_{B_{2}}, B_{3} V B$ and $B_{4} V$.
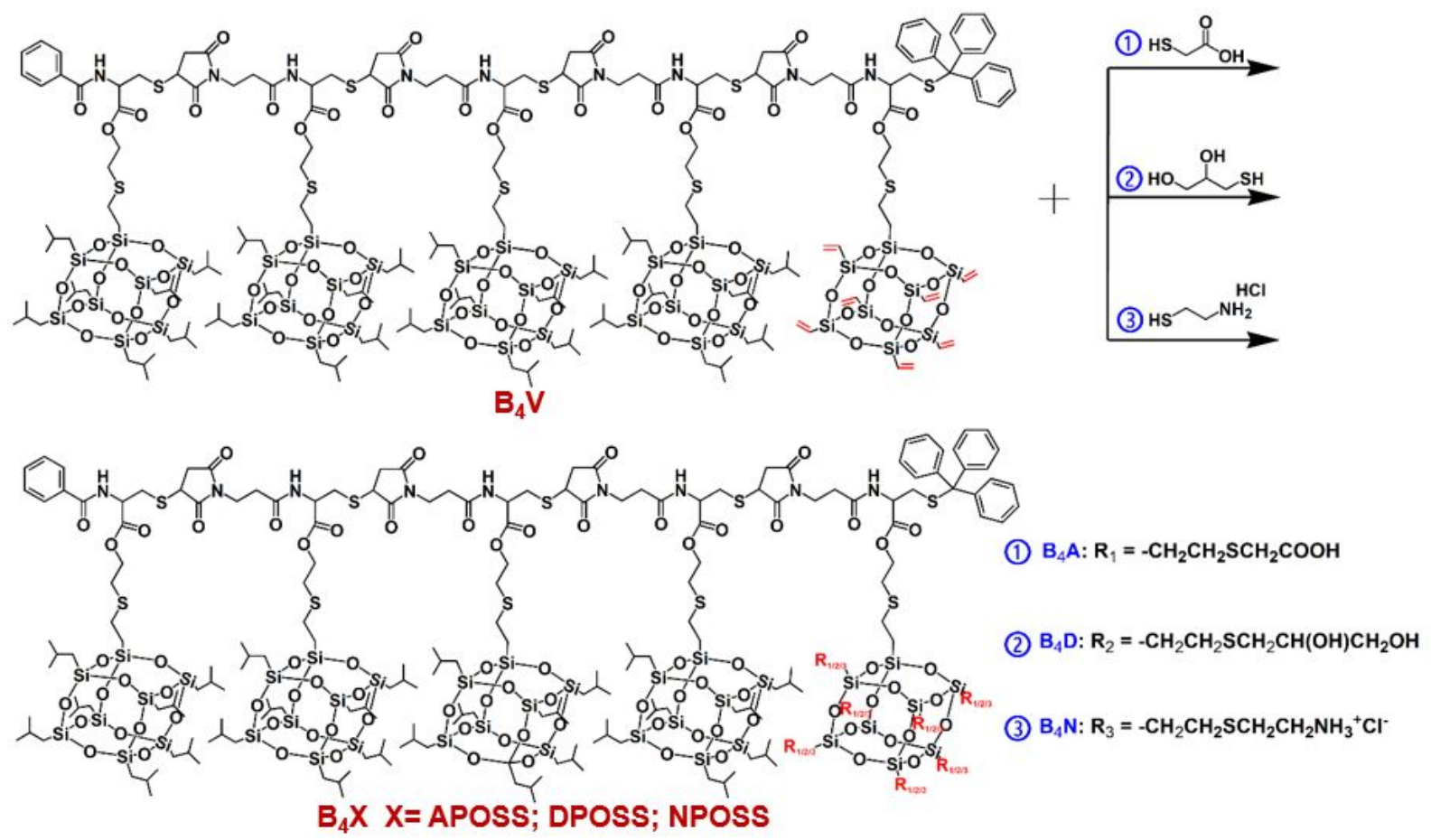

Scheme S3. Synthesis route of $\mathbf{B}_{\mathbf{4}} \mathbf{A}, \mathbf{B}_{\mathbf{4}} \mathbf{D}$ and $\mathbf{B}_{\mathbf{4}} \mathbf{N}$. 


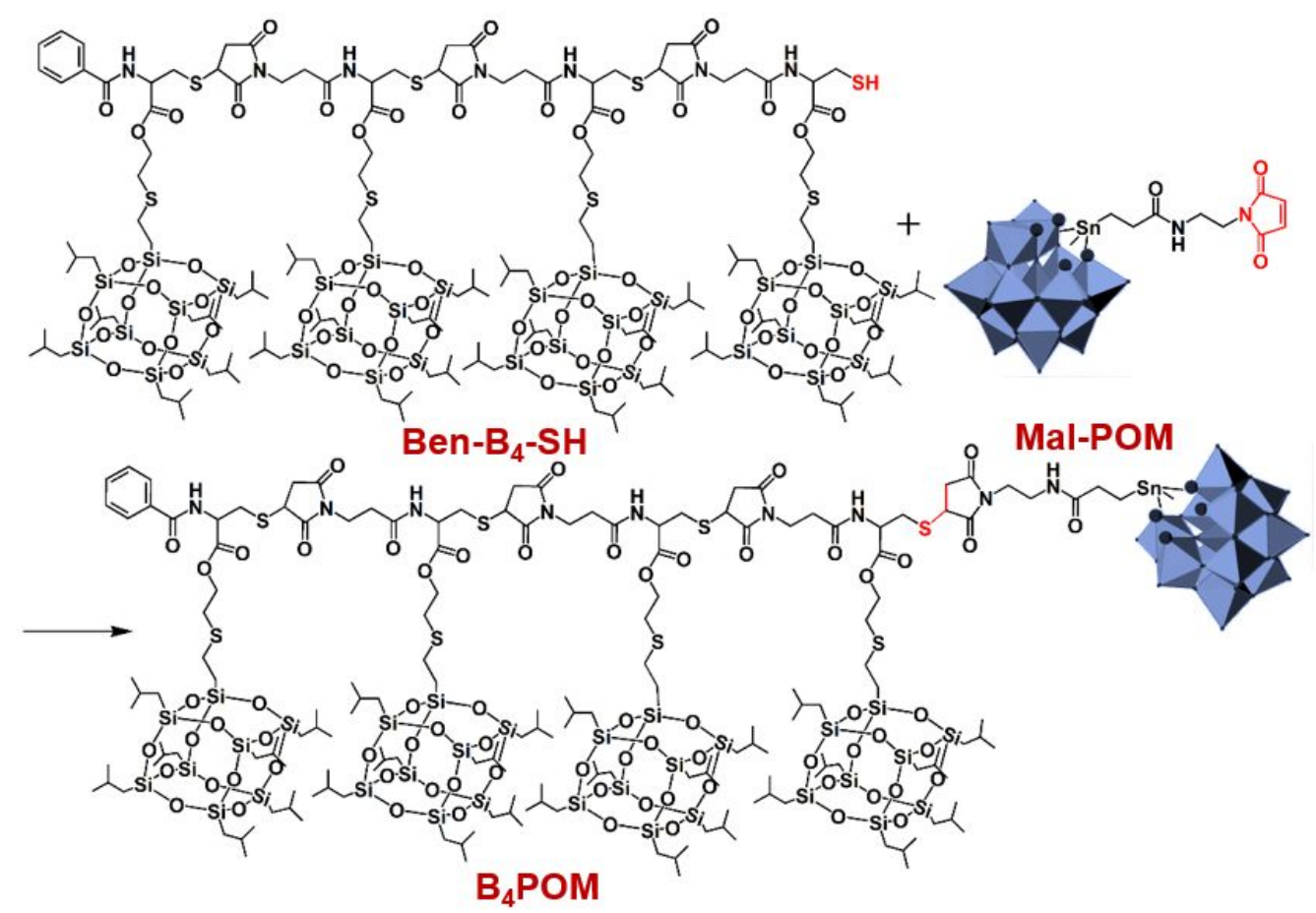

Scheme S4. Synthesis route of $\mathbf{B}_{\mathbf{4}} \mathbf{P O M}$.

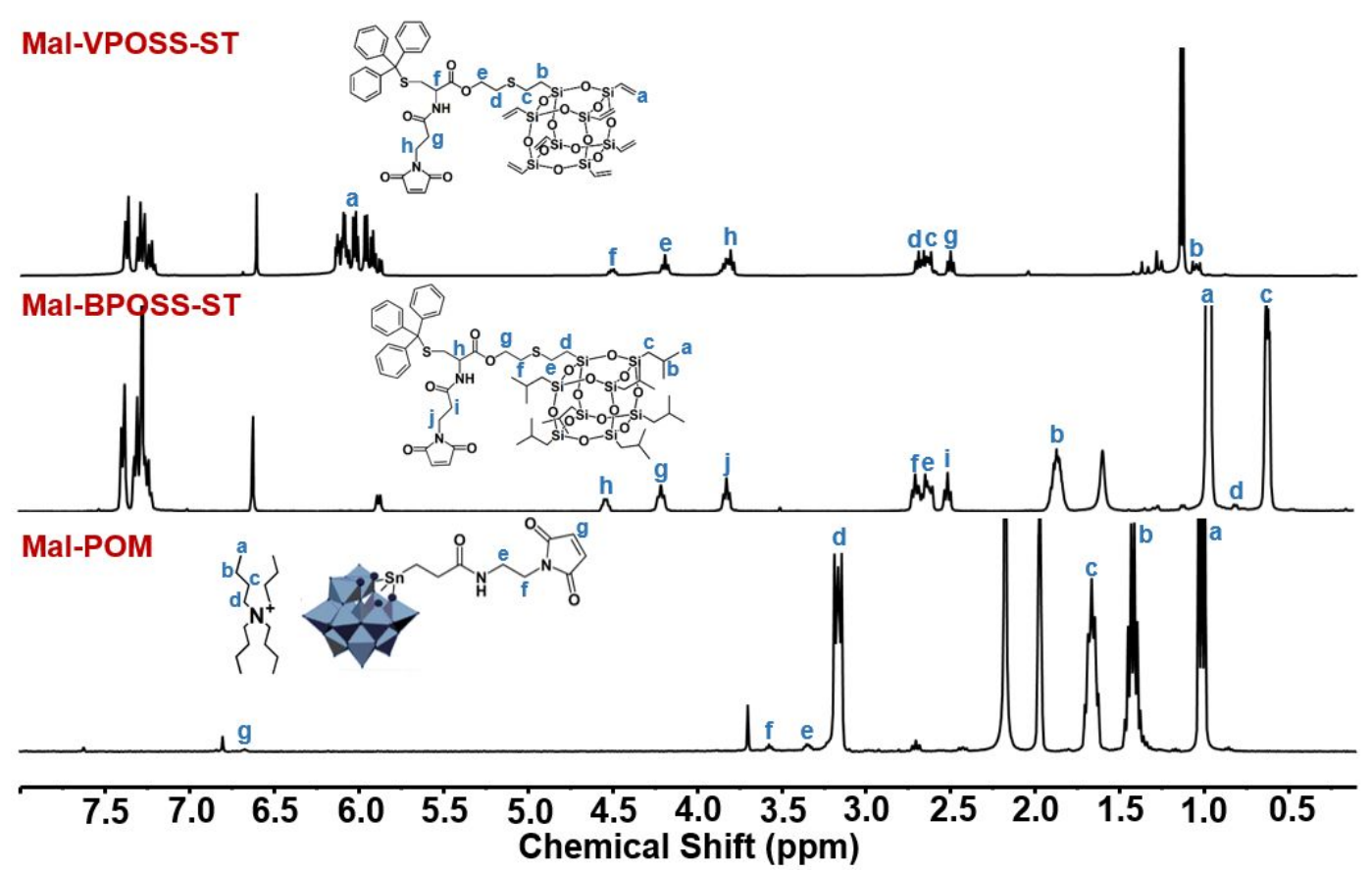

Figure S1. ${ }^{1} \mathrm{H}$ NMR spectra of Mal-VPOSS-ST, Mal-BPOSS-ST and Mal-POM. 


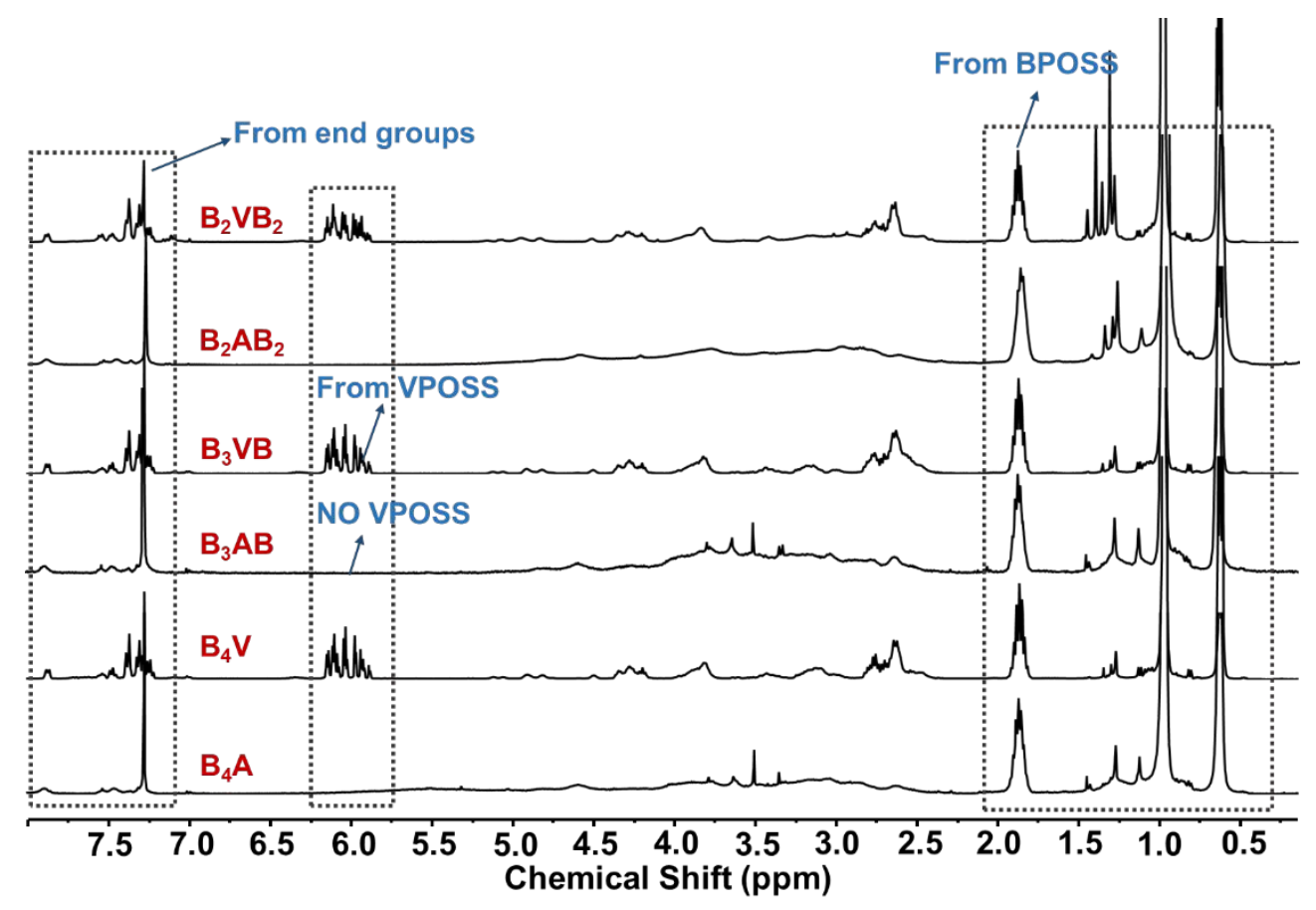

Figure S2. ${ }^{1} \mathrm{H}$ NMR spectra of $\mathbf{B}_{2} \mathbf{V B}_{2}, \mathbf{B}_{2} \mathbf{A} \mathbf{B}_{2}, \mathbf{B}_{3} \mathbf{V B}, \mathbf{B}_{3} \mathbf{A B}, \mathbf{B}_{\mathbf{4}} \mathbf{V}$ and $\mathbf{B}_{\mathbf{4}} \mathbf{A}$.

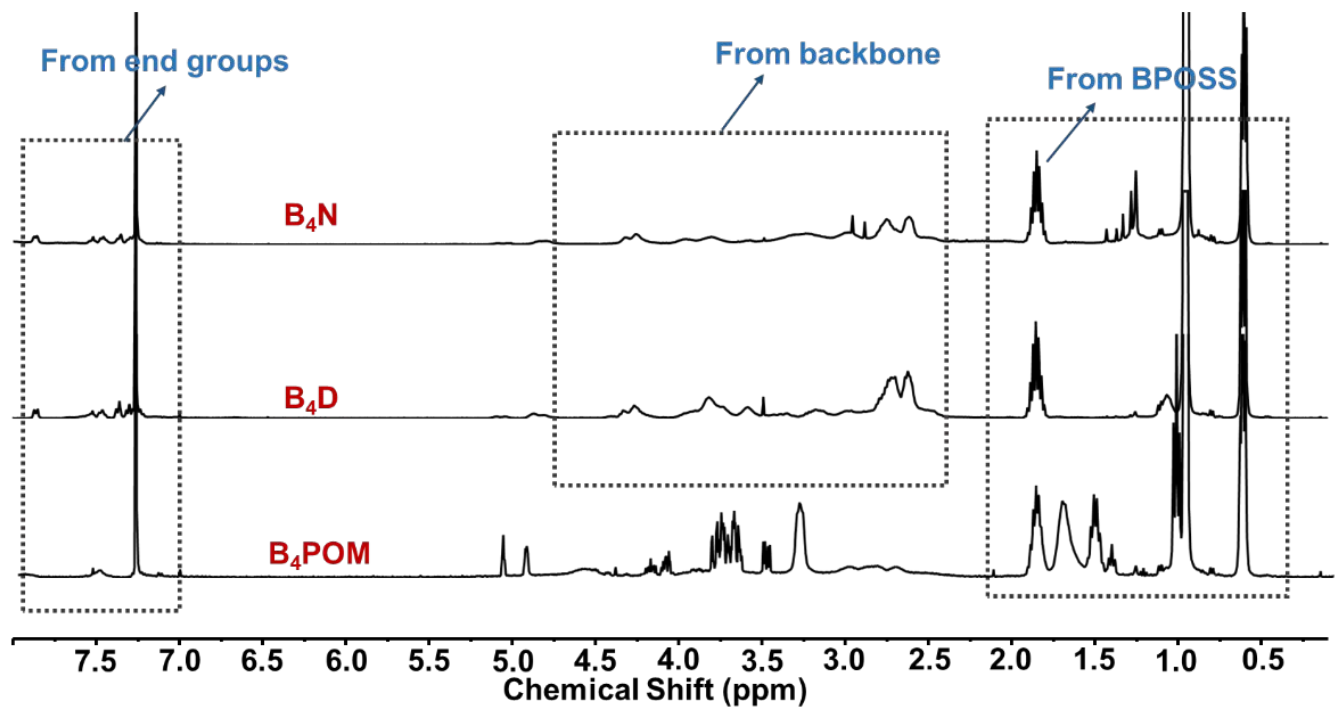

Figure S3. ${ }^{1} \mathrm{H}$ NMR spectra of $\mathbf{B}_{\mathbf{4}} \mathbf{N}, \mathbf{B}_{\mathbf{4}} \mathbf{D}$ and $\mathbf{B}_{\mathbf{4}} \mathbf{P O M}$. 


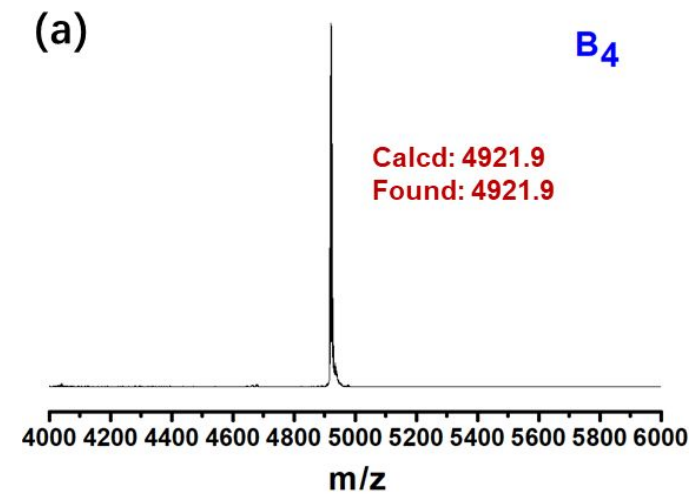

B 3 VB

(c)

5200540056005800600062006400660068007000 $\mathrm{m} / \mathrm{z}$ (b)

\section{$\mathrm{B}_{4} \mathrm{~V}$}

Calcd: $\mathbf{5 8 8 7 . 4}$

Found: $\mathbf{5 8 8 7 . 7}$ (d)

Calcd: 5887.4

Found: 5886.9

\section{$\mathrm{B}_{2} \mathrm{VB}_{2}$}

Calcd: $\mathbf{5 8 8 7 . 4}$

Found: 5887.9

Figure S4. MALDI-TOF MS of (a) $\mathbf{B}_{\mathbf{4}}$, (b) $\mathbf{B}_{\mathbf{4}} \mathbf{V}$, (c) $\mathbf{B}_{\mathbf{3}} \mathbf{V B}$ and (d) $\mathbf{B}_{\mathbf{2}} \mathbf{V B}_{\mathbf{2}}$.
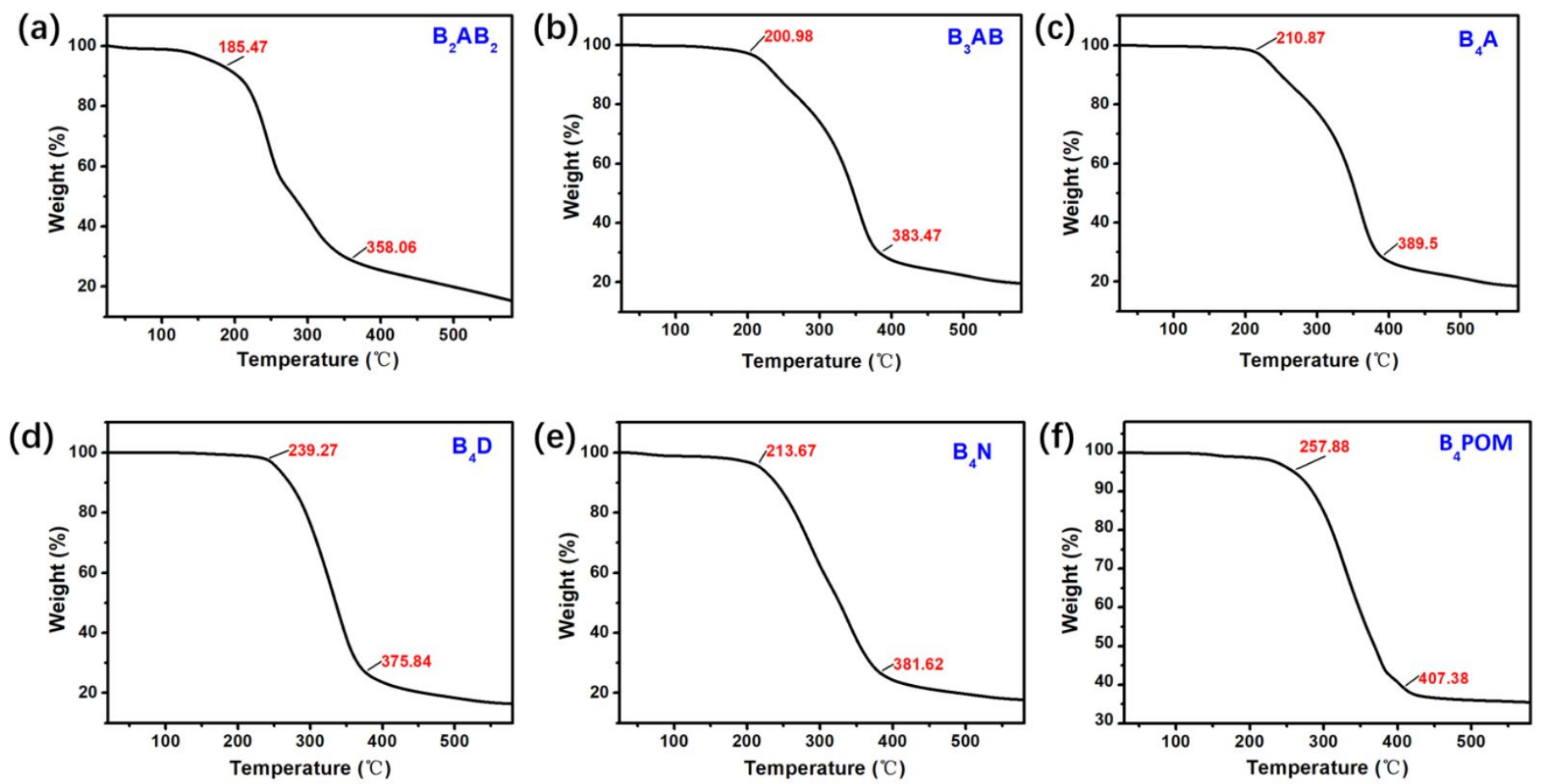

Figure S5. TGA analysis of (a) $\mathbf{B}_{2} \mathbf{A} \mathbf{B}_{2}$, (b) $\mathbf{B}_{3} \mathbf{A B}$, (c) $\mathbf{B}_{4} \mathbf{A}$, (d) $\mathbf{B}_{4} \mathbf{D}$, (e) $\mathbf{B}_{4} \mathbf{N}$ and (f) $\mathbf{B}_{4} \mathbf{P O M}$. 

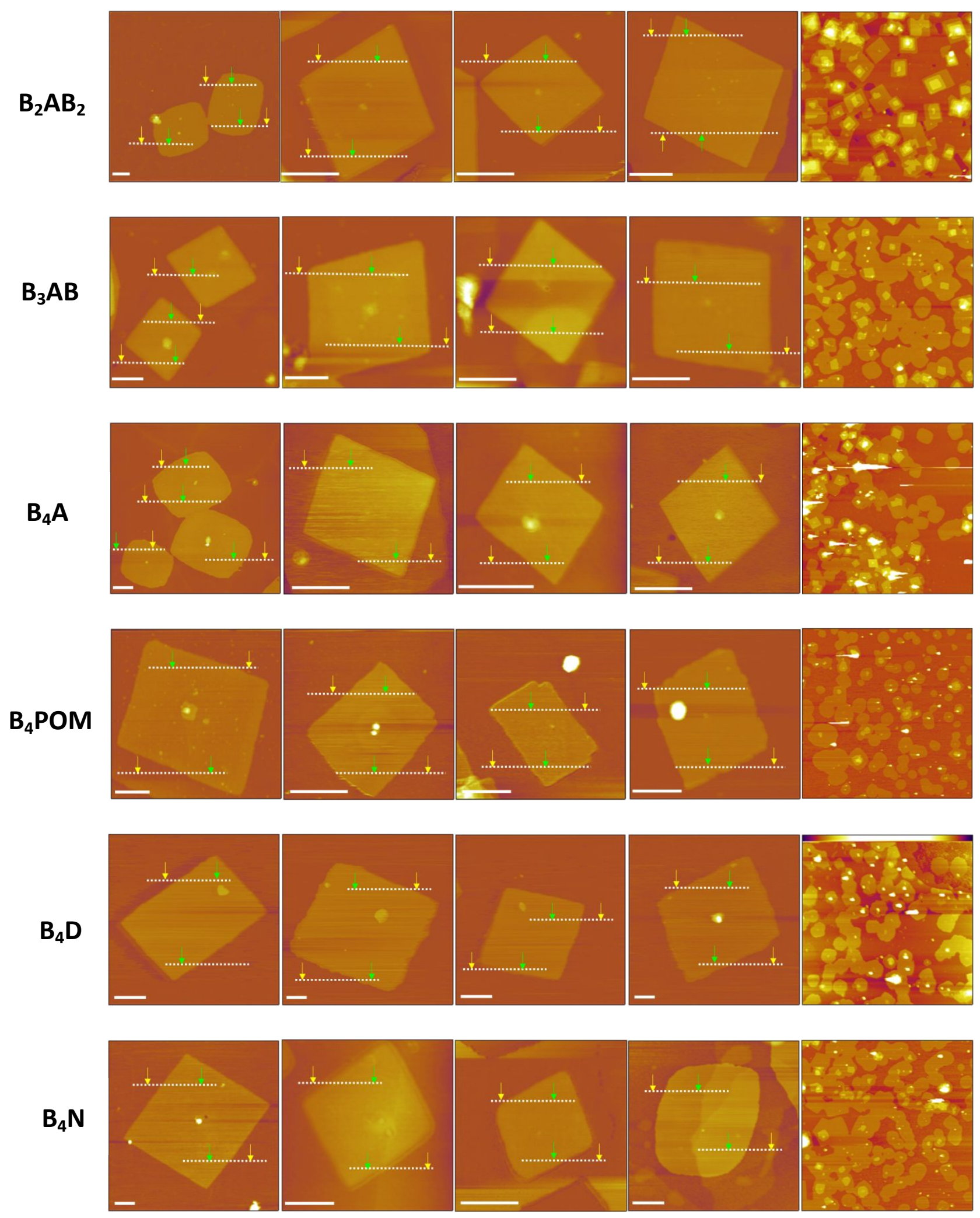

Figure S6. AFM images of giant molecules $\mathbf{B}_{\mathbf{2}} \mathbf{A} \mathbf{B}_{\mathbf{2}}, \mathbf{B}_{\mathbf{3}} \mathbf{A B}, \mathbf{B}_{\mathbf{4}} \mathbf{A}, \mathbf{B}_{\mathbf{4}} \mathbf{P O M}, \mathbf{B}_{\mathbf{4}} \mathbf{D}$ and $\mathbf{B}_{\mathbf{4}} \mathbf{N}$, (Scale bar $\left.=500 \mathrm{~nm}\right)$. The yellow arrow represents the base, and the green arrow represents the upper surface of the two-dimensional crystals. 


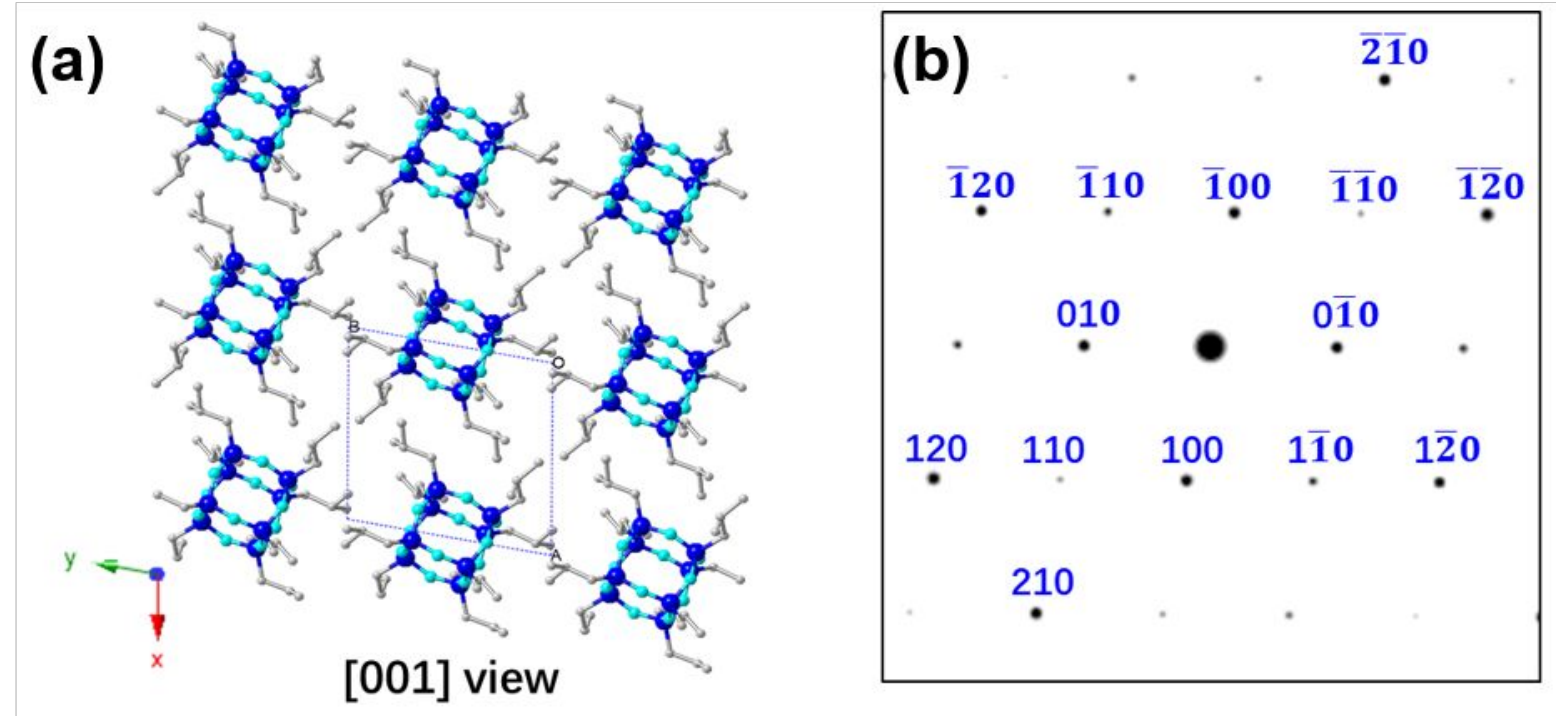

Figure S7. (a) [001] zone view of the crystal lattice model of T8 BPOSS, and (b) the corresponding simulated ED pattern with indices. Computer simulation was carried out with Accelrys Cerius2 software in our previous report. 

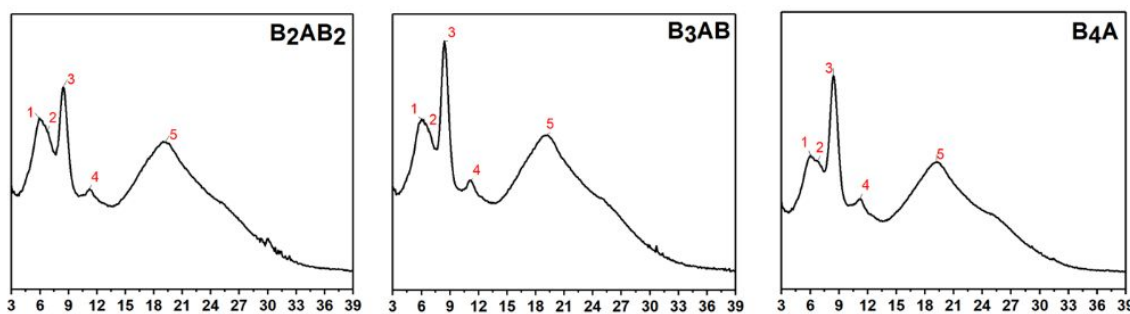

\begin{tabular}{|c|c|c|}
\hline \multicolumn{3}{|c|}{$\mathrm{B}_{2} \mathbf{A B}_{2}$} \\
\hline NO & $2 \theta$ & d-spacing \\
\hline 1 & 5.92 & 1.49 \\
\hline 2 & 6.75 & 1.31 \\
\hline 3 & 8.39 & 1.05 \\
\hline 4 & 11.18 & 0.79 \\
\hline 5 & 19.18 & 0.46 \\
\hline
\end{tabular}

\begin{tabular}{|c|c|c|}
\hline \multicolumn{3}{|c|}{$\mathrm{B}_{3} \mathrm{AB}$} \\
\hline NO & $2 \theta$ & d-spacing \\
\hline 1 & 6.02 & 1.46 \\
\hline 2 & 6.74 & 1.31 \\
\hline 3 & 8.39 & 1.05 \\
\hline 4 & 11.18 & 0.79 \\
\hline 5 & 19.18 & 0.46 \\
\hline
\end{tabular}

\begin{tabular}{|c|c|c|}
\hline \multicolumn{3}{|c|}{$\mathrm{B}_{4} \mathrm{~A}$} \\
\hline NO & $2 \theta$ & d-spacing \\
\hline 1 & 6.02 & 1.46 \\
\hline 2 & 6.74 & 1.31 \\
\hline 3 & 8.39 & 1.05 \\
\hline 4 & 11.18 & 0.79 \\
\hline 5 & 19.18 & 0.46 \\
\hline
\end{tabular}
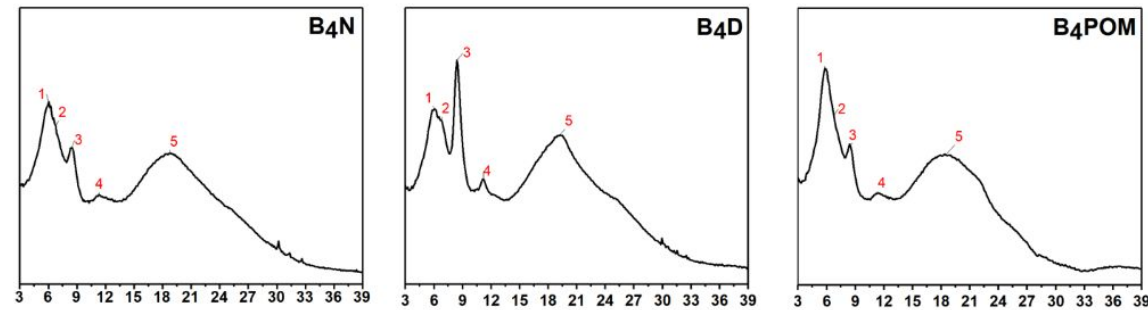

\begin{tabular}{|c|c|c|}
\hline \multicolumn{3}{|c|}{$\mathbf{B}_{4} \mathbf{N}$} \\
\hline NO & $2 \theta$ & d-spacing \\
\hline 1 & 6.03 & 1.46 \\
\hline 2 & 6.74 & 1.31 \\
\hline 3 & 8.39 & 1.05 \\
\hline 4 & 11.29 & 0.78 \\
\hline 5 & 18.76 & 0.47 \\
\hline
\end{tabular}

\begin{tabular}{|c|c|c|}
\hline \multicolumn{3}{|c|}{$\mathrm{B}_{4} \mathrm{D}$} \\
\hline NO & $2 \theta$ & d-spacing \\
\hline 1 & 6.02 & 1.46 \\
\hline 2 & 6.74 & 1.31 \\
\hline 3 & 8.39 & 1.05 \\
\hline 4 & 11.18 & 0.79 \\
\hline 5 & 19.18 & 0.46 \\
\hline
\end{tabular}

\begin{tabular}{|c|c|c|}
\hline \multicolumn{3}{|c|}{ B ${ }_{4}$ POM } \\
\hline NO & $2 \theta$ & d-spacing \\
\hline 1 & 5.82 & 1.52 \\
\hline 2 & 6.74 & 1.31 \\
\hline 3 & 8.39 & 1.05 \\
\hline 4 & 11.18 & 0.79 \\
\hline 5 & 18.75 & 0.47 \\
\hline
\end{tabular}

Figure S8. WAXD patterns of giant molecules $\mathbf{B}_{\mathbf{2}} \mathbf{A} \mathbf{B}_{2}, \mathbf{B}_{\mathbf{3}} \mathbf{A B}, \mathbf{B}_{\mathbf{4}} \mathbf{A}, \mathbf{B}_{\mathbf{4}} \mathbf{N}, \mathbf{B}_{\mathbf{4}} \mathbf{D}$ and $\mathbf{B}_{\mathbf{4}} \mathbf{P O M}$. 